\title{
Recent Progress of Geant4 Electromagnetic Physics and Readiness for the LHC Start
}

\author{
Vladimir Ivanchenko ${ }^{1}$, John Apostolakis \\ CERN, CH-1211 Geneve 23, Switzerland \\ E-mail: Vladimir.Ivantchenko@cern.ch, John.Apostolakis@cern.ch
}

\section{Andreas Schaelicke}

DESY, 15738 Zeuthen, Germany

E-mail: Andreas.Schaelicke@desy.de

\section{Laszlo Urban}

Geant4 Associates International Ltd, United Kingdom

E-mail: Laszlo.Urban@cern.ch

\section{Toshiyuki Toshito}

KEK, Tsukubo, Ibaraki 305-0801 Japan

E-mail: ttoshito@post.kek.jp

\section{Sabine Elles, Jean Jacquemier, Michel Maire}

LAPP, 74941 Annecy-le-vieux, France

E-mail: Sabine.Elles@lapp.in2p3.fr, Jean.Jacquemier@lapp.in2p3.fr, Michel.Maire@1app.in2p3.it

\section{Alexander Bagulya, Vladimir Grichine}

Lebedev Physics Institute, 119991Moscow, Russia

E-mail: Alexander.Bagulya@cern.ch, Vladimir.Grichine@cern.ch

\section{Alexei Bogdanov, Rostislav Kokoulin}

MEPhl, 115409 Moscow, Russia

E-mail: agbogdanov@mephi.ru, rpkokoulin@mephi.ru

Peter Gumplinger

TRIUMF, Vancouver, Canada

E-mail: gum@triumf.ca

The status of Geant4 electromagnetic (EM) physics models is presented, focusing on the models most relevant for collider HEP experiments, at LHC in particular.

XII Advanced Computing and Analysis Techniques in Physics Research Erice, Italy

3-7 November, 2008

$1 \quad$ Speaker 


\section{Introduction}

The EM package of the Geant4 toolkit [1,2] includes well established physics models and corresponding software. It has been used for many years in different applications, in particular, for Monte Carlo simulation of experiments in high energy physics [3-6]. Many efforts were concentrated on the preparing of the Geant4 EM physics package specifically for the LHC [7]. Recent improvements accomplished a better description of details of the EM physics. Models revised included those for single and multiple scattering, ionization at low and high energies, bremsstrahlung, scintillation and Cerenkov radiation. Validation has been performed against experimental data and theory. Highlights on new developments are presented below.

\section{Bremsstrahlung}

A review of the bremsstrahlung model was carried out, as presented in [8]. The density effect suppression has been refined according to the classical approach [9] and a new ultrarelativistic model with updated LMP effect has been created, which fit the experimental results [10] much better than the previous Geant4 model (Fig.1). The ultra-relativistic model is applicable for electrons and positrons above $1 \mathrm{GeV}$.

New Geant4 processes and models of bremsstrahlung and $\mathrm{e}^{+} \mathrm{e}^{-}$pair production by hadrons have been created using inheritance from existing Geant4 codes for muons [11]. Only modifications of the differential cross sections were required to take mass and spin differences between muons and hadrons into account. The cross sections of these hadron processes are small compared to the total hadronic cross section but a visible effect on the e/ $\pi$ separation for particles above $100 \mathrm{GeV}$ was observed in the CMS test-beam analysis [12]. The probability of a large pion energy deposition in CMS ECAL increased with increasing of the pion energy due to the high energy pion bremsstrahlung.

Fig.1 Bremsstrahlung spectrum for $287 \mathrm{GeV}$ electron beam on a $0.182 \mathrm{~mm}\left(\begin{array}{lll}4 \% & X_{0}\end{array}\right)$ Tantalum target: red histogram - Geant4 9.1, black histogram - Geant4 9.2 (new ultrarelativistic model), dotted line - LPM off, points - data of CERN experiment [10].

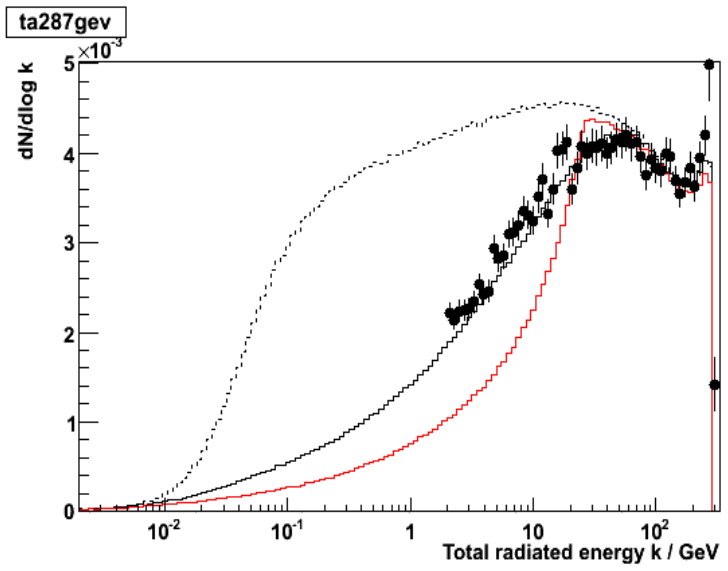

\section{Multiple scattering}

The Geant4 multiple scattering model (MSC) was under development for the last several years [7, 13] with a focus on providing the best balance between accuracy and CPU performance, which is a critical requirement for the LHC simulation. Recent modifications 
concentrated on implementing and tuning of MSC models specific to particle type, with electrons as our first goal. The description of the tail of the electron scattering distribution was improved and the single scattering model is applied to electrons in the vicinity of geometry boundaries. This allowed to double the default value of the parameter of MSC step limitation $F_{R}$ $=0.04$. Thus, by keeping practically the same CPU performance, a better agreement between simulation and data for the backscattering of electrons is achieved. In Fig.2 it is shown that both new MSC and the single scattering model [7] are close to the measurements. Note that a simulation using the single scattering process requires much more CPU, because of sampling of each elastic collision.

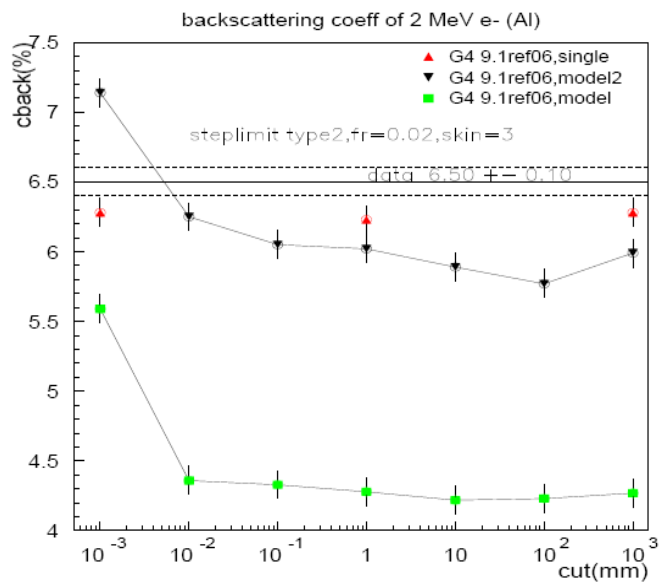

Fig.2 Electron backscattering simulation: green points - Geant4 9.1, black points - Geant4 9.2, red points - single scattering, horizontal line shows the data [14], dotted lines - data error.

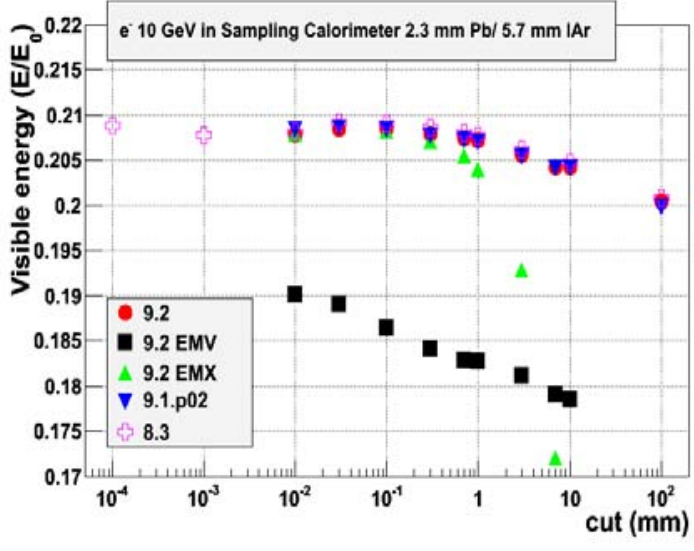

Fig.3 Simulation results for the ATLAS barrel type calorimeter response for different versions of Geant4: EMV - "fast" option (provides biased result), EMX - "ApplyCuts" option activated.

\section{Infrastructure upgrade and calorimeter response}

Various infrastructure upgrades of the EM software have been performed: the validation suite [7] was further extended; new helper classes were designed; extra options were introduced. In particular, a cubic spline interpolation of physics tables was provided, which is an alternative to the existing linear interpolation. Physics tables are created at initialisation time of Geant 4 to save computations during run time. These tables require significant memory. The accuracy of the interpolation depends on the number of bins. Usage of the spline interpolation method increased simulation precision while keeping the size of Geant 4 applications the same. In particular, this spline option enables a cut independent simulation of position and shape of the Bragg peak for proton or ion beams with a precision better than $0.1 \mathrm{~mm}$.

By default Geant4 production thresholds (cuts) are applied for processes of ionisation and bremsstrahlung only. The option "ApplyCuts" enables production thresholds for other EM processes (Compton scattering, photo-electric effect ...) speeding up simulation. However, it may affect the response of LHC calorimeters. The evolution of results as a function of the cut is controlled using the EM testing suite. The regression tests are performed between different Geant 4 versions. The ATLAS barrel type EM calorimeter is stable within $0.3 \%$ for the default Physics List (Fig.3). In case of the "ApplyCuts" option (EMX) the simulation is faster but 
shows a stronger dependence on the cut value. The response of the CMS-type crystal calorimeter (5x5 matrix) is less cut dependent (Fig.4), because practically all energy is delivered in the sensitive part of the detector. In such calorimeters the "fast" Physics List (EMV) provides similar results as the default one. The effect of the "ApplyCuts" option is seen for high cuts values as increasing of visible energy contrary to the sampling calorimeter results (Fig.3).

The transverse shape of EM showers can be characterized by the relative value of the energy deposition in the central crystal of the matrix (Fig.5). Because of the upgrade of MSC this value is reduced by $0.5 \%$ in comparison with previous Geant 4 versions and become closer to the data of the CMS test-beam measurements [6]. Other modifications, namely the new bremsstrahlung model and the spline interpolation, did not affect these results.

The performance benchmark for EM calorimeters is performed using the Linux SLC4 Geant4 installation at dedicated PC and static libraries (Table.1). The performance of the updated EM package is as good as of the previous Geant4 version 9.1 for the default EM physics configuration. About 20\% speedup is obtained if the "ApplyCuts" option is enabled.

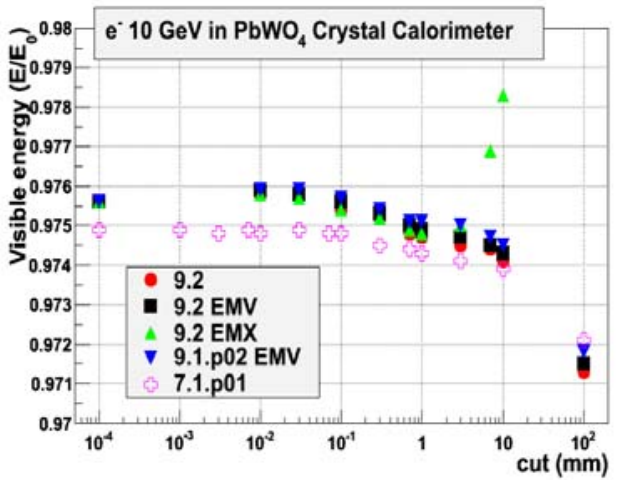

Fig.4 CMS-type EM calorimeter response with the different versions of Geant4: EMV - "fast" option provides biased result, EMX “ApplyCuts” option enabled.

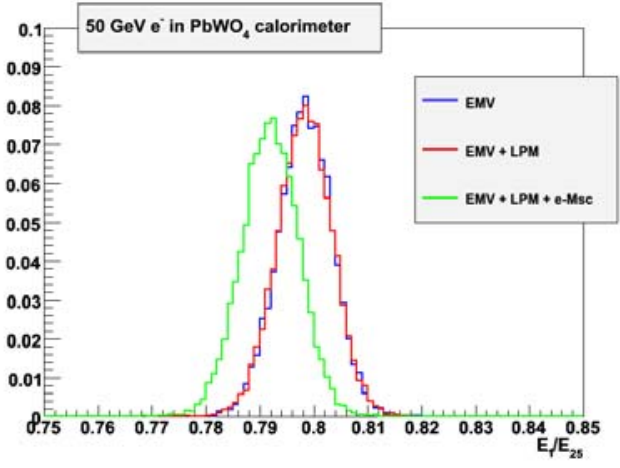

Fig.5 CMS-type EM calorimeter ratio of the energy depositions in the central tower and $5 \times 5$ matrix: blue - Geant4 9.1, red - new bremsstrahlung added, green - updated MSC.

Table 1 The results of the performance benchmark for EM calorimeters. For the EMV Physics Lists with the version 9.2 the "ApplyCuts" option is enabled. The results are normalized to the Geant4 version 8.3.

\begin{tabular}{|l|l|l|l|l|l|l|}
\hline $\begin{array}{l}\text { Geant4 } \\
\text { version }\end{array}$ & CMS & ATLAS & ATLAS & $\begin{array}{l}\text { CMS } \\
(\text { EMV })\end{array}$ & $\begin{array}{l}\text { ATLAS } \\
(\text { EMV) }\end{array}$ & $\begin{array}{l}\text { ATLAS } \\
(\text { EMV) }\end{array}$ \\
\hline Cut $(\mathrm{mm})$ & 0.7 & 0.7 & 0.02 & 0.7 & 0.7 & 0.02 \\
\hline 8.3 & 1.33 & 2.30 & 1.84 & 1.0 & 1.0 & 1.0 \\
\hline 9.0 & 1.21 & 2.05 & 1.65 & 0.92 & 0.93 & 0.94 \\
\hline $9.1 \mathrm{p} 02$ & 1.16 & 2.05 & 1.64 & 0.92 & 0.94 & 0.93 \\
\hline 9.2 & 1.11 & 1.84 & 1.49 & 0.72 & 0.81 & 0.87 \\
\hline
\end{tabular}

\section{Cerenkov radiation}

A review of the Cerenkov process has been performed. In the past a Cerenkov step limit was set only by user defined average maximum number of photons to be generated during the step. In the new version of the Cerenkov process the step can be limited by user specified 
maximum allowed change of the particle velocity during the step. Also a definite step limit occurs when the track drops below the Cerenkov threshold. Other modifications result in a more precise distribution of generated Cerenkov photons in space. Generation is performed after the sampling of MSC displacement of the end point of the step. Photons are not longer uniformly distributed along the step, but instead their number varies linearly with velocity change.

\section{Conclusions}

In recent years significant efforts have been spent on upgrades of Geant4 EM physics with focus on the simulation of LHC experiments. In this work the main improvements are highlighted which are included in the Geant4 release 9.2 (December 2008). Validation results with the EM testing suite confirm readiness of Geant4 EM simulation for the LHC start.

Acknowledgement This work has been supported in part by RFBR grant 09-02-91065 and CNRS grant PICS-4865.

\section{References}

[1] S. Agostinelli et al., Geant4: A simulation toolkit, Nucl. Instr. Meth. A 506 (2003) 250.

[2] J. Allison et al., Geant4 developments and applications, IEEE Trans. Nucl. Sci. 53 (2006) 270.

[3] S. Benerjee, Readiness of CMS simulation towards LHC start-up, J. Phys.Conf. Ser.119 (2008) 032006 .

[4] S. Benerjee et al., Validating Geant4 versions 7.1 and 8.3 against 6.1 for BaBar, J. Phys.Conf. Ser. 119 (2008) 032007.

[5] E. Barberio et al., Fast shower simulation in ATLAS calorimeter, J. Phys.Conf. Ser.119 (2008) 032008 .

[6] F. Cossutti, The simulation of the CMS electromagnetic calorimeter, J. Phys.Conf. Ser.119 (2008) 032015.

[7] J. Apostolakis et al., The performance of Geant4 Standard EM package for LHC and other applications, J. Phys.Conf. Ser.119 (2008) 032004.

[8] A. Schaelicke et al., Improved description of bremsstrahlung for high-energy electrons in Geant4, in proceedings of IEEE NSS conference, Dresden, Germany, 19-25 October 2008, NSS N37-1.

[9] M. L. Ter-Mikaelian, High-energy electromagnetic processes in condensed media, Wiley, 1972.

[10] H. D. Hansen et al., Landau-Pomeranchuk-Migdal effect for multi-hundred GeV electrons, Phys.Rev. D 69 (2004) 032001.

[11] A. Bogdanov et al., Geant4 simulation of production and interaction of muons, IEEE Trans. Nucl. Sci. 53 (2006) 513.

[12] S. Piperov, Calorimeter simulation with hadrons in CMS, in proceedings of IEEE NSS conference, Dresden, Germany, 19 - 25 October 2008, NSS N37-8.

[13] L. Urban, A multiple scattering model in Geant4, Preprint CERN-OPEN-2006-077.

[14] M. Rosenstein et al., Electron depth-dose distribution measurements in finite polysterene slabs, J.Appl. Phys. 43 (1972) 3191. 